\title{
STATISTICAL ANALYSIS OF FAILURE TIME DATA WITH MISSING INFORMATION
}

\author{
Ping Chen
}

Dr. (Tony) Jianguo Sun, Dissertation Supervisor

\begin{abstract}
Failure time data arise in many fields and can involve different types of censoring structures and missing information. We consider three cases: right-censored data with missing censoring indicators, clustered current status data, and clustered interval-censored data.

Chapter 2 discusses regression analysis of right-censored failure time data with missing censoring indicators and presents an efficient estimation procedure based on the EM algorithm. The simulation study performed indicates that the proposed methodology performs well for practical situations. An illustrative example from a breast cancer clinical trial is provided.

Chapter 3 discusses regression analysis of clustered current status data. For inference, a Cox frailty model and a two-step EM algorithm are presented. A simulation study was conducted for the evaluation of the proposed methodology and indicates that the approach performs well for practical situations. An illustrative example from a tumorigenicity experiment is provided.

Chapter 4 generalizes the study of Chapter 3 to clustered interval-censored data. Chapter 5 discusses some directions for future research.
\end{abstract}

